\title{
Linear Quadratic Stochastic Optimal Control of Forward Backward Stochastic Control System Associated with Lévy Process
}

\author{
Hong Huang, ${ }^{1,2}$ Xiangrong Wang, ${ }^{1}$ Ting $\mathrm{Hou}^{3}$ and $\mathrm{Lu} \mathrm{Xu}^{4}$ \\ ${ }^{1}$ Institute of Financial Engineering, College of Mathematics and Systems Science, Shandong University of Science and Technology, \\ Qingdao 266590, China \\ ${ }^{2}$ Institute of Financial Engineering, Shandong Women's University, Jinan 250300, China \\ ${ }^{3}$ College of Mathematics and Systems Science, Shandong University of Science and Technology, Qingdao 266590, China \\ ${ }^{4}$ School of Statistics and Management, Shanghai University of Finance and Economics, Shanghai 200433, China
}

Correspondence should be addressed to Xiangrong Wang; xrwang2000@126.com

Received 5 April 2017; Accepted 17 August 2017; Published 25 September 2017

Academic Editor: Zhongwei Lin

Copyright (C) 2017 Hong Huang et al. This is an open access article distributed under the Creative Commons Attribution License, which permits unrestricted use, distribution, and reproduction in any medium, provided the original work is properly cited.

This paper analyzes one kind of linear quadratic (LQ) stochastic control problem of forward backward stochastic control system associated with Lévy process. We obtain the explicit form of the optimal control, then prove it to be unique, and get the linear feedback regulator by introducing one kind of generalized Riccati equation. Finally, we discuss the solvability of the generalized Riccati equation, and its existence and uniqueness of the solutions are proved in a special case.

\section{Introduction}

LQ stochastic optimal control is a kind of special optimal control problem, which not only can be used to model many linear optimal problems practically, but also can reasonably be used to approach and solve many nonlinear problems. In 1962, Kushner [1] firstly established a forward random stochastic LQ model with a dynamic programming method and Wonham [2] firstly studied a LQ stochastic optimal control problem by introducing a Riccati equation in 1968. Then a lot of works have been done for forward or backward stochastic LQ control problems, the corresponding Riccati equation, and its application in finance, such as Li and Zhang [3], Ma and Hou [4], Liu et al. [5], Wang et al. [6], and Shen and Wang [7]. In 2003, Wang et al. [8] discussed a special kind of forward backward stochastic LQ problem and got the existence and uniqueness of the optimal control for the control system. Subsequently, Wu [9] extended this conclusion to the fully coupled forward backward stochastic LQ problem.

The optimal control problem with random jumps was first considered by Boel and Varaiya [10]; in this case, the control system is often described by Brownian motion and Poisson processes. On the basis of proving the existence and uniqueness of solutions of a kind of forward backward stochastic differential equation with Poisson jumps (FBSDEP), Wu and Wang [11] got the explicit form of the optimal control for LQ stochastic control problem where the state variable was described by a stochastic differential equation with a Poisson process (SDEP). In 2009, Shi and $\mathrm{Wu}$ [12] extended $\mathrm{Wu}$ and Wang's results in [11] to a fully coupled LQ stochastic control problem of forward backward stochastic control system with Poisson jumps. Moreover, Lin and Zhang [13] considered the $H_{\infty}$ control problem for linear stochastic systems driven by both Brownian motion and Poisson jumps. In 2016, Li et al. [14] studied a stochastic differential equations driven by GBrownian motion and got the existence and uniqueness of the solution for these equations.

In 2000, Nualart and Schoutens [15] introduced a class of Lévy processes with exponential moments satisfying some conditions. Using these exponential moments and the standard orthogonalization process, they constructed a series of orthogonal normal martingales called Teugels martingale. And they also proved a martingale representation 
theorem associated with Teugels martingale. In the next year, Nualart and Schoutens [16] considered a backward stochastic differential equation (BSDE) driven by Teugels martingale and proved the existence and uniqueness theory of this BSDE. In 2003, Bahlali et al. [17] studied a BSDE driven by Teugels martingale and an independent Brownian motion; they got the existence, uniqueness, and comparison of solutions for these equations, having a Lipschitz or locally Lipschitz coefficient. El Otmani [18] considered a kind of generalized BSDE (GBSDE) associated with Teugels martingale and Brownian motion associated with a pure jump-independent Lévy process. They got the existence and uniqueness theory of this GBSDE when the coefficient verifies some conditions of Lipschitz. More results about BSDE associated with Teugels martingale can be found in the theses of El Otmani [19], Ren and Fan [20], Tang and Zhang [21], and Huang and Wang [22]. On the basis of these results, in 2008, Mitsui and Tabata [23] studied a LQ regulation stochastic control problem with Lévy process and obtained the optimal control for the nonhomogeneous case. In [24], Tang and Wu considered the following LQ stochastic control problem in a given finite horizon $[s, T]$ with Lévy process:

$$
\begin{aligned}
d x(t)= & {[A(\omega, t) x(t)+B(\omega, t) u(t)] d t } \\
& +\sum_{i=1}^{\infty}\left[C^{i}(\omega, t) x\left(t^{-}\right)+D^{i}(\omega, t) u(t)\right] d H^{i}(t) \\
x(s)=\xi &
\end{aligned}
$$

and the cost function was

$$
\begin{aligned}
& J(s, \xi, u(\cdot))=E^{s}\left[\int _ { s } ^ { T } \left(x^{\tau}(t) Q(\omega, t) x(t)\right.\right. \\
& \left.\left.\quad+u^{\tau}(t) R(\omega, t) u(t)\right) d t+x^{\tau}(T) H(\omega) x(T)\right] .
\end{aligned}
$$

They show that the solvability of one kind of generalized Riccati equation is sufficient to the well-posedness of this LQ problem and proved the existence of the optimal control.

In this paper, we consider one kind of LQ stochastic control problem where the controlled system is driven by a fully coupled linear forward backward stochastic differential equation associated with Lévy process (FBSDEL).

$$
\begin{aligned}
& d x_{t}=\left[A(\omega, t) x_{t}+B(\omega, t) u_{t}-L(\omega, t) y_{t}\right] d t \\
& +\left[C(\omega, t) x_{t}+D(\omega, t) u_{t}\right] d B_{t} \\
& +\sum_{i=1}^{\infty}\left[E^{i}(\omega, t) x_{t_{-}}+F^{i}(\omega, t) u_{t}\right] d H_{t}^{i} \\
& -d y_{t}=\left[A^{\tau}(\omega, t) y_{t}+C^{\tau}(\omega, t) z_{t}+\sum_{i=1}^{\infty} E^{i, \tau}(\omega, t) r_{t}^{i}\right. \\
& \left.+R(\omega, t) x_{t}\right] d t-z_{t} d B_{t}-\sum_{i=1}^{\infty} r_{t}^{i} d H_{t}^{i}
\end{aligned}
$$

$$
\begin{aligned}
& x_{0}=a, \\
& y_{T}=Q(\omega) x_{T},
\end{aligned}
$$

where $\left(x_{t}, y_{t}, z_{t}, r_{t}^{i}\right)$ are $\mathscr{F}_{t}$-adapted stochastic processes taking values in $R^{n} \times R^{n} \times R^{n} \times l^{2}\left(R^{n}\right)$ and $u(\cdot)$ is $\mathscr{F}_{t}$-adapted stochastic process called admissible control process. Assume the control process set $U=R^{k}$ and define the admissible control set as follows:

$$
\begin{aligned}
U_{a d} & =\left\{u(\cdot) \in M^{2}\left(0, T ; R^{k}\right) ; u_{t} \in U, 0 \leq t\right. \\
\leq T & \text {, a.e., a.s. }\} .
\end{aligned}
$$

The cost functional we considered is

$$
\begin{aligned}
& J(u)=\frac{1}{2} E\left[\int _ { 0 } ^ { T } \left(\left\langle R(\omega, t) x_{t}, x_{t}\right\rangle+\left\langle N(\omega, t) u_{t}, u_{t}\right\rangle\right.\right. \\
& \left.\left.\quad+\left\langle L(\omega, t) y_{t}, y_{t}\right\rangle\right) d t+\left\langle Q(\omega) x_{T}, x_{T}\right\rangle\right] .
\end{aligned}
$$

And the optimal control problem is to find $\bar{u}_{t} \in U_{a d}$, such that

$$
J(\bar{u}(\cdot))=\inf _{u(\cdot)} J(u(\cdot)) .
$$

Note that (3) is a fully coupled FBSDEL. In 2012, Pereira and Shamarova [25] firstly considered this kind of FBSDEL, obtained a solution to this FBSDEL via a partial integrodifferential equation, and proved the uniqueness. Under some monotonicity assumptions, Baghery et al. [26] proved the existence and uniqueness of solutions of fully coupled FBSDEL and then obtained the existence of an openloop Nash equilibrium point for nonzero sum stochastic differential games by using this result. Based on [25], Wang and Huang [27] got the maximum principle for forward backward stochastic control system driven by Lévy process; then they discussed a kind of LQ stochastic control problem of forward backward stochastic control system and got a necessary condition for the optimal control.

We extend the result of Shi and Wu [12] to the fully coupled linear forward backward stochastic control system driven by Brownian motion and an independent Teugels martingale. Since Teugels martingale is more complex than the Poisson process, we also need more general formula about càdlàg semimartingale. The rest of this paper is organized as follows. In Section 2, we provide a list of notations and results of the existence and uniqueness of solutions of fully coupled FBSDEL. In Section 3, we prove the existence and uniqueness of the optimal control of LQ stochastic control problem (6) and give the linear feedback regulator for the optimal control by the solution of a kind of generalized matrix-valued Riccati equation when assuming the coefficient matrices are deterministic. In Section 4, the solvability of this kind of matrix-valued Riccati equation is discussed.

\section{Preliminaries and Notations}

Let $\left(\Omega, \mathscr{F}_{t}, P\right)$ be a complete probability space satisfying the usual conditions; $\mathscr{F}_{t}$ is a right continuous increasing family of 
complete sub- $\sigma$-algebra which is generated by the following two mutually independent processes: a one-dimensional standard Brownian motion $\left\{B_{t}\right\}_{0 \leq t \leq T}$ and an $R$-valued Lévy process $\left\{L_{t}\right\}_{0 \leq t \leq T}$ with a standard Lévy measure $\nu$ satisfy

(i) $\int_{R}\left(1 \wedge x^{2}\right) \nu(d x)<\infty$,

(ii) $\int_{(-\varepsilon, \varepsilon)^{c}} e^{\lambda|x|} \nu(d x)<\infty$, for every $\varepsilon>0$ and for some $\lambda>0$.

Naluart and Schoutens denoted Teugels martingale associated with the Lévy process $\left\{L_{t}\right\}_{0 \leq t \leq T}$ by $\left\{H_{t}^{i}\right\}_{i=1}^{\infty}$, and $H_{t}^{i}$ is given by

$$
H_{t}^{i}=c_{i, i} Y_{t}^{i}+c_{i, i-1} Y_{t}^{i-1}+c_{i, i-2} Y_{t}^{i-2}+\cdots+c_{i, 1} Y_{t}^{1}
$$

where $Y_{t}^{i}=L_{t}^{i}-E\left[L_{t}^{i}\right]$ is the compensated power-jump process of order $i$ and $L_{t}^{i}$ is power-jump processes:

$$
L_{t}^{i}= \begin{cases}L_{t}, & i=1, \\ \sum_{0<s \leq t}\left(\Delta L_{s}\right)^{i}, & i \geq 2,\end{cases}
$$

Coefficients $c_{i, k}$ correspond to orthonormalization of the polynomials $1, x, x^{2}, \ldots$ with respect the measure $\mu(d x)=$ $v(d x)+\sigma^{2} \delta_{0}(d x)$. Please refer to Naluart and Schoutens [15] for more details about Teugels martingale.

Introduce the following notations adopted in this paper:

$\langle A, B\rangle=\operatorname{tr}\left(A B^{T}\right)$ : the inner product in $R^{n \times m}, \forall A, B \in$ $R^{n \times m}$

$|\alpha|=\sqrt{\langle\alpha, \alpha\rangle}$ : the norm in $R^{n}, \forall \alpha \in R^{n}$

$L^{2}(\Omega, H)$ : the space of $H$-valued $\mathscr{F}_{T}$-measurable random variable $\xi$ satisfies $E|\xi|^{2}<\infty$

$M^{2}(0, T ; H)$ : the space of $H$-valued $\mathscr{F}_{t}$-measurable process $\phi(\cdot)=\{\phi(t, \omega):(t, \omega) \in[0, T] \times \Omega\}$ satisfies $E \int_{0}^{T}\left|\phi_{t}\right|^{2} d t<\infty$

$l^{2}(H)$ : the space of $H$-valued $\left\{f^{i}\right\}_{i \geq 1}$ satisfies $\sum_{i=1}^{\infty}\left|f^{i}\right|^{2}<\infty$

$l^{2}(0, T ; H)$ : the space of $l^{2}(H)$-valued $\mathscr{F}_{t}$-measurable processes satisfies $E \int_{0}^{T} \sum_{i=1}^{\infty}\left|f_{t}^{i}\right|^{2} d t<\infty$

$S^{2}(0, T ; H)$ : the space of $H$-valued $\mathscr{F}_{t}$-measurable càdlàg process $f(\cdot)=\{f(t, \omega):(t, \omega) \in[0, T] \times \Omega\}$ satisfies $E \sup _{0 \leq t \leq T}\left|f_{t}\right|^{2} d t<\infty$.

For notational brevity, we set

$$
\begin{aligned}
M^{2}(0, T)= & M^{2}\left(0, T ; R^{n}\right) \times M^{2}\left(0, T ; R^{n}\right) \\
& \times M^{2}\left(0, T ; R^{n}\right) \times l^{2}\left(0, T ; R^{n}\right) .
\end{aligned}
$$

Next, consider the following fully coupled FBSDEL

$$
\begin{aligned}
d x_{t}= & b\left(t, x_{t}, y_{t}, z_{t}, r_{t}\right) d t+\sum_{i=1}^{d} \sigma^{i}\left(t, x_{t}, y_{t}, z_{t}, r_{t}\right) d B_{t}^{i} \\
& +\sum_{i=1}^{\infty} g^{i}\left(t, x_{t-}, y_{t-}, z_{t}, r_{t}\right) d H_{t}^{i} \\
-d y_{t}= & f\left(t, x_{t}, y_{t}, z_{t}, r_{t}\right) d t-\sum_{i=1}^{d} z_{t}^{i} d B_{t}^{i}-\sum_{i=1}^{\infty} r_{t}^{i} d H_{t}^{i} \\
x_{0}= & a, \\
y_{T}= & \Phi\left(x_{T}\right),
\end{aligned}
$$

where $b: \Omega \times[0, T] \times R^{n} \times R^{m} \times R^{m \times d} \times l^{2}\left(R^{m}\right) \rightarrow R^{n}, \sigma:$ $\Omega \times[0, T] \times R^{n} \times R^{m} \times R^{m \times d} \times l^{2}\left(R^{m}\right) \rightarrow R^{n \times d}, g: \Omega \times[0, T] \times$ $R^{n} \times R^{m} \times R^{m \times d} \times l^{2}\left(R^{m}\right) \rightarrow l^{2}\left(R^{n}\right), f: \Omega \times[0, T] \times R^{n} \times R^{m} \times$ $R^{m \times d} \times l^{2}\left(R^{m}\right) \rightarrow R^{m}$.

For a given $m \times n$ full rank matrix $G$, set

$$
\begin{aligned}
\lambda & =\left(\begin{array}{c}
x \\
y \\
z
\end{array}\right), \\
A(t, \lambda, r) & =\left(\begin{array}{c}
-G^{\tau} f(t, \lambda, r) \\
G b(t, \lambda, r) \\
G \sigma(t, \lambda, r)
\end{array}\right) .
\end{aligned}
$$

Assumption 1. (i) $b, \sigma, g$, and $f$ are uniformly Lipschitz continuous with respect to $(x, y, z, r)$.

(ii) For each $(\omega, t) \in \Omega \times[0, T], l(\omega, t, 0,0,0,0) \in M^{2}(0, T)$ and $g(\omega, t, 0,0,0,0) \in H^{2}\left(l^{2}\right)$, where $l=b, \sigma, f$, respectively.

(iii) $\Phi(\cdot)$ is uniformly Lipschitz continuous with respect to $x$ and $\forall x, \Phi(x) \in L^{2}\left(\Omega, F_{T}, P\right)$.

Assumption 2.

$$
\begin{aligned}
& \left\langle A\left(t, \lambda_{1}, r_{1}\right)-A\left(t, \lambda_{2}, r_{2}\right), \lambda_{1}-\lambda_{2}\right\rangle+\sum_{i=1}^{\infty}\left\langle G \widehat{g^{i}}, \widehat{r^{i}}\right\rangle \\
& \leq-\beta_{1}|G \widehat{x}|^{2} \\
& \quad-\beta_{2}\left(\left|G^{\tau} \hat{y}\right|^{2}+\left|G^{\tau} \widehat{z}\right|^{2}+\sum_{i=1}^{\infty}\left\|G^{\tau} \widehat{r^{i}}\right\|^{2}\right) ; \\
& \left\langle\Phi\left(x_{1}\right)-\Phi\left(x_{2}\right), G\left(x_{1}-x_{2}\right)\right\rangle \geq \mu_{1}|G \widehat{x}|^{2},
\end{aligned}
$$

where $\lambda_{1}=\left(x_{1}, y_{1}, z_{1}\right), \lambda_{2}=\left(x_{2}, y_{2}, z_{2}\right), \hat{x}=x_{1}-x_{2}, \hat{y}=$ $y_{1}-y_{2}, \widehat{z}=z_{1}-z_{2}, \widehat{g}^{i}=g^{i}\left(t, \lambda_{1}, r_{1}\right)-g^{i}\left(t, \lambda_{2}, r_{2}\right), \widehat{r^{i}}=r_{1}^{i}-r_{2}^{i}$, and $\beta_{1}, \beta_{2}, \mu_{1}$ are nonnegative constants with $\beta_{1}+\beta_{2}>0$ and $\beta_{2}+\mu_{1}>0$. Moreover, we have $\beta_{1}>0, \mu_{1}>0$ (resp., $\beta_{2}>0$ ) when $m>n$ (resp., $n>m$ ).

Lemma 3 (existence and uniqueness theorem of FBSDEL [25]). Under Assumptions 1 and 2, FBSDEL (10) admits a unique solution in $M^{2}(0, T)$. 
In the following sections we also need the more general Ito's formula about a càdlàg semimartingales.

Lemma 4 (Ito's formula [27]). Let $X=\left\{X_{t}: t \in[0, T]\right\}$ be càdlàg semimartingales, denote $[X]=\left\{[X]_{t}: t \in[0, T]\right\}$ as the quadratic variation process, $F$ is a $\mathscr{C}^{2}$ real valued function, then $F(X)$ is also a semimartingales, and the following Ito's formula holds

$$
\begin{aligned}
F\left(X_{t}\right)= & F\left(X_{0}\right)+\int_{0}^{t} F^{\prime}\left(X_{s-}\right) d X_{s} \\
& +\frac{1}{2} \int_{0}^{t} F^{\prime \prime}\left(X_{s}\right) d[X]_{s}^{\mathscr{C}} \\
& +\sum_{0<s \leq t}\left\{F\left(X_{s}\right)-F\left(X_{s^{-}}\right)-F^{\prime}\left(X_{s-}\right) \Delta X_{s}\right\} .
\end{aligned}
$$

where $[X]^{\mathscr{C}}$ is the continuous part of $[X]$.

In particular, when $F(X)=X^{2}$ and $F(X)=X_{t} Y_{t}$, where $X, Y$ are two càdlàg semimartingales, we get

$$
\begin{aligned}
X_{t}^{2}= & X_{0}^{2}+\int_{0}^{t} 2 X_{s-} d X_{s}+\int_{0}^{t} d[X]_{s} \\
X_{t} Y_{t}= & X_{0} Y_{0}+\int_{0}^{t} X_{s-} d Y_{s}+\int_{0}^{t} Y_{s-} d X_{s} \\
& +\int_{0}^{t} d[X, Y]_{s} .
\end{aligned}
$$

Here $[X, Y]$ is the quadratic covariation of $X, Y$.

\section{Linear Quadratic Stochastic Optimal Control Problem}

Let us consider the LQ stochastic optimal control problem (6). First of all, we give some necessary explanations for the coefficients in the system:

$A(\omega, t), C(\omega, t), E^{i}(\omega, t),(i=1,2,3, \ldots) \in R^{n \times n}$, $B(\omega, t), D(\omega, t)$, and $F^{i}(\omega, t),(i=1,2,3, \ldots) \in R^{n \times k}$ are all bounded progressively measurable matrix-valued processes. $R(\omega, t), L(\omega, t) \in R^{n \times n}$ are nonnegative symmetric bounded progressively measurable matrix-valued processes, and $N(\omega, t)$ is a positive bounded $k \times k$ progressively measurable matrix-valued process; the inverse is $N^{-1}(\omega, t)$, which is also bounded. $Q(\omega)$ is a $\mathscr{F}_{t}$-adapted nonnegative symmetric bounded matrix-valued random variable.

For a given admissible control $u(\cdot) \in U_{a d}$, under assumptions of the coefficients above, we can verify that FBSDEL (3) satisfies Assumptions 1 and 2. Therefore, there exists a unique solution $\left(x_{t}^{u}, y_{t}^{u}, z_{t}^{u}, r_{t}^{u}\right) \in M^{2}(0, T)$ satisfying the control system (3) from Lemma 3.

Then we get the explicit form of the optimal control $u_{t}$ for the LQ stochastic optimal control problem (6).
Theorem 5. There exists a unique optimal control $u_{t}$ for $L Q$ stochastic optimal control problem (6), and $u_{t}$ is given by the following equation.

$$
\begin{aligned}
u_{t}= & -N^{-1}(\omega, t) \\
& \cdot\left(B^{\tau}(\omega, t) y_{t}+D^{\tau}(\omega, t) z_{t}+\sum_{i=1}^{\infty} F^{i, \tau}(\omega, t) r_{t}^{i}\right) .
\end{aligned}
$$

Proof. As we know, for a given admissible control $u_{t}$, the control system (15) has a unique solution $\left(x_{t}, y_{t}, z_{t}, r_{t}\right) \in$ $M^{2}(0, T)$.

Existence. For any admissible control $v_{t}$, assume the corresponding trajectory is $\left(x_{t}^{v}, y_{t}^{v}, z_{t}^{v}, r_{t}^{v}\right) \in M^{2}(0, T)$; then

$$
\begin{aligned}
& J\left(v_{t}\right)-J\left(u_{t}\right)=\frac{1}{2} \\
& +E\left[\int _ { 0 } ^ { T } \left(\left\langle R(\omega, t)\left(x_{t}^{v}-x_{t}\right), x_{t}^{v}-x_{t}\right\rangle\right.\right. \\
& +\left\langle 2 R(\omega, t) x_{t}, x_{t}^{v}-x_{t}\right\rangle \\
& +\left\langle N(\omega, t)\left(v_{t}-u_{t}\right), v_{t}-u_{t}\right\rangle \\
& +\left\langle 2 N(\omega, t) u_{t}, v_{t}-u_{t}\right\rangle \\
& +\left\langle L(\omega, t)\left(y_{t}^{v}-y_{t}\right), y_{t}^{v}-y_{t}\right\rangle \\
& \left.+\left\langle 2 L(\omega, t) y_{t}, y_{t}^{v}-y_{t}\right\rangle\right) d t \\
& +\left\langle Q(\omega)\left(x_{T}^{v}-x_{T}\right), x_{T}^{v}-x_{T}\right\rangle+\left\langle 2 Q(\omega) x_{T}, x_{T}^{v}\right. \\
& \left.\left.\quad-x_{T}\right\rangle\right] .
\end{aligned}
$$

Applying Ito's formula to $\left\langle x_{t}^{v}-x_{t}, y_{t}\right\rangle$ we have

$$
\begin{aligned}
E & \left\langle x_{T}^{v}-x_{T}, y_{T}\right\rangle=E \int_{0}^{T}\left(\left\langle-R(\omega, t) x_{t}, x_{t}^{v}-x_{t}\right\rangle\right. \\
& +\left\langle B^{\tau}(\omega, t) y_{t}, v_{t}-u_{t}\right\rangle-\left\langle L(\omega, t) y_{t}, y_{t}^{v}-y_{t}\right\rangle \\
& +\left\langle D^{\tau}(\omega, t) z_{t}, v_{t}-u_{t}\right\rangle \\
& \left.+\sum_{i=1}^{\infty}\left\langle F^{i, \tau}(\omega, t) r_{t}^{i}, v_{t}-u_{t}\right\rangle\right) d t .
\end{aligned}
$$

Since $R(\omega, t), L(\omega, t)$, and $Q(\omega)$ are nonnegative and $N(\omega, t)$ is positive, we can get

$$
\begin{aligned}
& J\left(v_{t}\right)-J\left(u_{t}\right) \\
& \quad \geq E \int_{0}^{T}\left[\left\langle\left(B^{\tau}(\omega, t) y_{t}+D^{\tau}(\omega, t) z_{t}+\sum_{i=1}^{\infty} F^{i, \tau}(\omega, t) r_{t}^{i}\right),\right.\right.
\end{aligned}
$$




$$
\begin{aligned}
& \left.\left.v_{t}-u_{t}\right\rangle+\left\langle N(\omega, t) u_{t}, v_{t}-u_{t}\right\rangle\right]=E \int_{0}^{T}\left\langle\left( B^{\tau}(\omega, t) y_{t}\right.\right. \\
& \left.\left.+D^{\tau}(\omega, t) z_{t}+\sum_{i=1}^{\infty} F^{i, \tau}(\omega, t) r_{t}^{i}\right), v_{t}-u_{t}\right\rangle-\langle N(\omega, t) \\
& \cdot N^{-1}(\omega, t)\left(B^{\tau}(\omega, t) y_{t}+D^{\tau}(\omega, t) z_{t}+\sum_{i=1}^{\infty} F^{i, \tau}(\omega, t) r_{t}^{i}\right), \\
& \left.v_{t}-u_{t}\right\rangle=0 .
\end{aligned}
$$

Then the admissible control $u_{t}$ defined by (15) is the optimal control of LQ stochastic control problem (6).

Unique. Assume admissible control $u_{t}^{1}$ is an optimal control; the corresponding trajectories are $\left(x_{t}^{1}, y_{t}^{1}, z_{t}^{1}, r_{t}^{1}\right)$ and $u_{t}^{2}$ is another optimal control; the corresponding trajectories are $\left(x_{t}^{2}, y_{t}^{2}, z_{t}^{2}, r_{t}^{2}\right)$. So the trajectories corresponding to $\left(u_{t}^{1}+u_{t}^{2}\right) / 2$ are

$$
\left(\frac{x_{t}^{1}+x_{t}^{2}}{2}, \frac{y_{t}^{1}+y_{t}^{2}}{2}, \frac{z_{t}^{1}+z_{t}^{2}}{2}, \frac{r_{t}^{1}+r_{t}^{2}}{2}\right),
$$

and the trajectories corresponding to $\left(u_{t}^{1}-u_{t}^{2}\right) / 2$ are

$$
\left(\frac{x_{t}^{1}-x_{t}^{2}}{2}, \frac{y_{t}^{1}-y_{t}^{2}}{2}, \frac{z_{t}^{1}-z_{t}^{2}}{2}, \frac{r_{t}^{1}-r_{t}^{2}}{2}\right) \text {. }
$$

Since $u_{t}^{1}$ and $u_{t}^{2}$ are both optimal controls, $N(\omega, t)$ is positive, and $R(\omega, t), L(\omega, t), Q(\omega)$ are nonnegative, we have

$$
\begin{aligned}
J\left(u_{t}^{1}\right)=J\left(u_{t}^{2}\right)=\alpha \geq 0 \\
2 \alpha=J\left(u_{t}^{1}\right)+J\left(u_{t}^{2}\right)=2 J\left(\frac{u_{t}^{1}+u_{t}^{2}}{2}\right) \\
+E \int_{0}^{T}\left(\left\langle R(\omega, t) \frac{x_{t}^{1}-x_{t}^{2}}{2}, \frac{x_{t}^{1}-x_{t}^{2}}{2}\right\rangle\right. \\
+\left\langle N(\omega, t) \frac{u_{t}^{1}-u_{t}^{2}}{2}, \frac{u_{t}^{1}-u_{t}^{2}}{2}\right\rangle \\
\left.+\left\langle L(\omega, t) \frac{y_{t}^{1}-y_{t}^{2}}{2}, \frac{y_{t}^{1}-y_{t}^{2}}{2}\right\rangle\right) d t \\
+E\left\langle Q(\omega) \frac{x_{T}^{1}-x_{T}^{2}}{2}, \frac{x_{T}^{1}-x_{T}^{2}}{2}\right\rangle \geq 2 J\left(\frac{u_{t}^{1}+u_{t}^{2}}{2}\right) \\
+E \int_{0}^{T}\left\langle N(\omega, t) \frac{u_{t}^{1}-u_{t}^{2}}{2}, \frac{u_{t}^{1}-u_{t}^{2}}{2}\right\rangle d t \geq 2 \alpha \\
+\frac{\delta}{2} E \int_{0}^{T}\left|u_{t}^{1}-u_{t}^{2}\right|^{2} d t .
\end{aligned}
$$

Here $\delta$ is a constant and $\delta>0$; then

$$
E \int_{0}^{T}\left|u_{t}^{1}-u_{t}^{2}\right|^{2} d t \leq 0
$$

hence, $u_{t}^{1}=u_{t}^{2}$ in $M^{2}\left(0, T ; R^{k}\right)$.
Assume $A(\omega, t), B(\omega, t), C(\omega, t), D(\omega, t), E(\omega, t), F(\omega, t)$, $R(\omega, t), N(\omega, t), L(\omega, t)$, and $Q(\omega)$ are all deterministic matrices, denoted as $A_{t}, B_{t}, C_{t}, D_{t}, E_{t}, F_{t}, R_{t}, N_{t}, L_{t}$, and $Q$ for convenience. Introducing the following generalized $n \times n$ matrix-valued Riccati equation (23), $t \in[0, T], i=1,2, \ldots$

$$
\begin{aligned}
-\dot{K}_{t}= & A_{t}^{\tau} K_{t}+K_{t} A_{t}+C_{t}^{\tau} M_{t}+\sum_{i=1}^{\infty}\left(E_{t}^{i}\right)^{\tau} Y_{t}^{i} \\
& -K_{t}\left(L_{t}^{\tau}+B_{t} N_{t}^{-1} B_{t}^{\tau}\right) K_{t}-K_{t} B_{t} N_{t}^{-1} D_{t}^{\tau} M_{t} \\
& -\sum_{i=1}^{\infty} K_{t} B_{t} N_{t}^{-1}\left(F_{t}^{i}\right)^{\tau} Y_{t}^{i}+R_{t} \\
M_{t}= & K_{t} C_{t}-K_{t} D_{t} N_{t}^{-1} B_{t}^{\tau} K_{t}-K_{t} D_{t} N_{t}^{-1} D_{t} M_{t} \\
& -\sum_{i=1}^{\infty} K_{t} D_{t} N_{t}^{-1}\left(F_{t}^{i}\right)^{\tau} Y_{t}^{i} \\
Y_{t}^{i}= & K_{t} E_{t}^{i}-K_{t} F_{t}^{i} N_{t}^{-1} B_{t}^{\tau} K_{t}-K_{t} F_{t}^{i} N_{t}^{-1} D_{t}^{\tau} M_{t} \\
& -\sum_{i=1}^{\infty} K_{t} F_{t}^{i} N_{t}^{-1}\left(F_{t}^{i}\right)^{\tau} Y_{t}^{i} \\
K_{T}= & Q ;
\end{aligned}
$$

Then we can get the following conclusions.

Theorem 6. Suppose the generalized matrix-valued Riccati equation (23) has solution $\left(K_{t}, M_{t}, Y_{t}^{i}\right)$ for all $t \in[0, T]$; then the optimal linear feedback regulator for $L Q$ stochastic optimal control problem (6) is

$$
u_{t}=-N_{t}^{-1}\left[B_{t}^{\tau} K_{t}+D_{t}^{\tau} M_{t}+\sum_{i=1}^{\infty}\left(F_{t}^{i}\right)^{\tau} Y_{t}^{i}\right] x_{t}
$$

and the optimal value function is

$$
J\left(u_{t}\right)=\frac{1}{2}\left\langle K_{0} a, a\right\rangle .
$$

Proof. If $\left(K_{t}, M_{t}, Y_{t}^{i}\right)$ is the solution of the matrix-valued Riccati equation (23), then we can check that the solution of (6) $\left(x_{t}, y_{t}, z_{t}, r_{t}^{i}\right)$ satisfies

$$
\begin{aligned}
& y_{t}=K_{t} x_{t}, \\
& z_{t}=M_{t} x_{t}, \\
& r_{t}^{i}=Y_{t}^{i} x_{t} .
\end{aligned}
$$

As we have proved that the optimal control has the form of (15), take (26) into (15); then the optimal control can be written by

$$
u_{t}=-N_{t}^{-1}\left[B_{t}^{\tau} K_{t}+D_{t}^{\tau} M_{t}+\sum_{i=1}^{\infty}\left(F_{t}^{i}\right)^{\tau} Y_{t}^{i}\right] x_{t}
$$


For the optimal value function, using Ito's formula to $\left\langle x_{t}, y_{t}\right\rangle$, then

$$
\begin{aligned}
& E \int_{0}^{T}\left\langle R_{t} x_{t}, x_{t}\right\rangle d t+E \int_{0}^{T}\left\langle L_{t} y_{t}, y_{t}\right\rangle d t+\left\langle Q x_{T}, x_{T}\right\rangle \\
& \quad-\langle K a, a\rangle \\
& =E \int_{0}^{T}\left\langle y_{t}, B_{t} u_{t}\right\rangle d t+E \int_{0}^{T}\left\langle z_{t}, D_{t} u_{t}\right\rangle d t \\
& \quad+E \int_{0}^{T} \sum_{i=1}^{\infty}\left\langle r_{t}^{i}, F_{t}^{i} u_{t}\right\rangle d t .
\end{aligned}
$$

On the other hand, from the relationship of $u$ and $\left(x_{t}, y_{t}, z_{t}, r_{t}^{i}\right)$, we can verify that

$$
\begin{aligned}
& E \int_{0}^{T}\left\langle y_{t}, B_{t} u_{t}\right\rangle d t+E \int_{0}^{T}\left\langle z_{t}, D_{t} u_{t}\right\rangle d t \\
& \quad+E \int_{0}^{T} \sum_{i=1}^{\infty}\left\langle r_{t}^{i}, F_{t}^{i} u_{t}\right\rangle d t=-E \int_{0}^{T}\left\langle N_{t} u_{t}, u_{t}\right\rangle d t
\end{aligned}
$$

and then

$$
\begin{aligned}
& E \int_{0}^{T}\left\langle R_{t} x_{t}, x_{t}\right\rangle d t+E \int_{0}^{T}\left\langle L_{t} y_{t}, y_{t}\right\rangle d t \\
& \quad+E \int_{0}^{T}\left\langle N_{t} u_{t}, u_{t}\right\rangle d t+\left\langle Q x_{T}, x_{T}\right\rangle=\langle K a, a\rangle .
\end{aligned}
$$

By the definition of cost function $J(\cdot)(5)$, we prove that the optimal value function is

$$
J\left(u_{t}\right)=\frac{1}{2}\left\langle K_{0} a, a\right\rangle .
$$

Now consider a special case of stochastic LQ control problem when $L(\omega, t)=0$, and the control system is reduced to

$$
\begin{aligned}
d x_{t}= & \left(A(\omega, t) x_{t}+B(\omega, t) u_{t}\right) d t \\
& +\left(C(\omega, t) x_{t}+D(\omega, t) u_{t}\right) d B_{t} \\
& +\sum_{i=1}^{\infty}\left(E^{i}(\omega, t) x_{t_{-}}+F^{i}(\omega, t) u_{t}\right) d H_{t}^{i} \\
x_{0}= & a .
\end{aligned}
$$

The cost functional now is

$$
\begin{aligned}
& J(u)=\frac{1}{2} E\left[\int_{0}^{T}\left\langle R(\omega, t) x_{t}, x_{t}\right\rangle+\left\langle N(\omega, t) u_{t}, u_{t}\right\rangle\right. \\
& \left.\quad+\left\langle Q(\omega) x_{T}, x_{T}\right\rangle\right] .
\end{aligned}
$$

Remark 7. Comparing the LQ stochastic optimal control system (32) and control system (1) which was considered in [22] by Tang and $\mathrm{Wu}$, we know that control system (1) is a special case of control system (32) when $C(\omega, t)=D(\omega, t)=$ 0 .
We can get the following Corollary 8 easily from Theorem 5 .

Corollary 8. There exists a unique optimal control for LQ stochastic optimal control problem (32)-(33), and

$$
\begin{aligned}
u_{t} & =-N^{-1}(\omega, t) \\
& \cdot\left[B^{\tau}(\omega, t) y_{t}+D^{\tau}(\omega, t) z_{t}+\sum_{i=1}^{\infty} F^{i, \tau}(\omega, t) r_{t}^{i}\right],
\end{aligned}
$$

where the $\left(y_{t}, z_{t}, r_{t}\right)$ is the solution of the following BSDE driven by Lévy process.

$$
\begin{aligned}
& -d y_{t}=\left[A^{\tau}(\omega, t) y_{t}+C^{\tau}(\omega, t) z_{t}+\sum_{i=1}^{\infty} E^{i, \tau}(\omega, t) r_{t}^{i}\right. \\
& \left.+R(\omega, t) x_{t}\right] d t-z_{t} d B_{t}-\sum_{i=1}^{\infty} r_{t}^{i} d H_{t}^{i} \\
& y_{T}=Q(\omega) x_{T} .
\end{aligned}
$$

Assume $A(\omega, t), B(\omega, t), C(\omega, t), D(\omega, t), E(\omega, t), F(\omega, t)$, $R(\omega, t), N(\omega, t)$, and $Q(\omega)$ are all deterministic; then Riccati equation (23) changes to

$$
\begin{aligned}
-\dot{K}_{t}= & A_{t}^{\tau} K_{t}+K_{t} A_{t}+C_{t}^{\tau} M_{t} \\
& +\sum_{i=1}^{\infty}\left(E_{t}^{i}\right)^{\tau} Y_{t}^{i}-K_{t} B_{t} N_{t}^{-1} B_{t}^{\tau} K_{t} \\
& -K_{t} B_{t} N_{t}^{-1} D_{t}^{\tau} M_{t}-\sum_{i=1}^{\infty} K_{t} B_{t} N_{t}^{-1}\left(F_{t}^{i}\right)^{\tau} Y_{t}^{i} \\
& +R_{t} \\
M_{t}= & K_{t} C_{t}-K_{t} D_{t} N_{t}^{-1} B_{t}^{\tau} K_{t}-K_{t} D_{t} N_{t}^{-1} D_{t} M_{t} \\
& -\sum_{i=1}^{\infty} K_{t} D_{t} N_{t}^{-1}\left(F_{t}^{i}\right)^{\tau} Y_{t}^{i} \\
Y_{t}^{i}= & K_{t} E_{t}^{i}-K_{t} F_{t}^{i} N_{t}^{-1} B_{t}^{\tau} K_{t}-K_{t} F_{t}^{i} N_{t}^{-1} D_{t}^{\tau} M_{t} \\
& -\sum_{i=1}^{\infty} K_{t} F_{t}^{i} N_{t}^{-1}\left(F_{t}^{i}\right)^{\tau} Y_{t}^{i} \\
K_{T}= & Q .
\end{aligned}
$$

Then from Theorem 6 we can get Corollary 9.

Corollary 9. For LQ stochastic optimal control problem (32)(33), if, for all $t \in[0, T]$, there exist matrices $\left(K_{t}, M_{t}, Y_{t}^{i}\right)$ satisfying (36), then the optimal linear feedback regulator is

$$
u_{t}=-N_{t}^{-1}\left[B_{t}^{\tau} K_{t}+D_{t}^{\tau} M_{t}+\sum_{i=1}^{\infty}\left(F_{t}^{i}\right)^{\tau} Y_{t}^{i}\right] x_{t}
$$

and the optimal value function is

$$
J\left(u_{t}\right)=\frac{1}{2}\left\langle K_{0} a, a\right\rangle .
$$




\section{Solvability of the Generalized Riccati Equation}

From the discussion of the previous section, we can see that the key to get the optimal linear feedback regulator for LQ stochastic optimal control problem is the solvability of the generalized Riccati equation (23). But (23) is so complicated that we cannot prove its existence and uniqueness at this moment. Using technique introduced by Shi and Wu [12], we only discuss a special case: $D_{t}=0$; in this case Riccati equation (23) becomes

$$
\begin{aligned}
-\dot{K}_{t}= & A_{t}^{\tau} K_{t}+K_{t} A_{t}+C_{t}^{\tau} M_{t}+\sum_{i=1}^{\infty}\left(E_{t}^{i}\right)^{\tau} Y_{t}^{i} \\
& -K_{t}\left(L_{t}^{\tau}+B_{t} N_{t}^{-1} B_{t}^{\tau}\right) K_{t} \\
& -\sum_{i=1}^{\infty} K_{t} B_{t} N_{t}^{-1}\left(F_{t}^{i}\right)^{\tau} Y_{t}^{i}+R_{t} \\
Y_{t}^{i}= & K_{t} E_{t}^{i}-K_{t} F_{t}^{i} N_{t}^{-1} B_{t}^{\tau} K_{t}-\sum_{i=1}^{\infty} K_{t} F_{t}^{i} N_{t}^{-1}\left(F_{t}^{i}\right)^{\tau} Y_{t}^{i}, \\
M_{t}= & K_{t} C_{t}, \\
K_{T}= & Q,
\end{aligned}
$$

Equivalently, consider the following equation:

$$
\begin{aligned}
&- \dot{K}_{t}=A_{t}^{\tau} K_{t}+K_{t} A_{t}+C_{t}^{\tau} K_{t} C_{t}-K_{t}\left(L_{t}^{\tau}+B_{t} N_{t}^{-1} B_{t}^{\tau}\right) \\
& \cdot K_{t}+R_{t}+\sum_{i=1}^{\infty}\left(E_{t}^{i}\right)^{\tau}\left[I_{n}+K_{t} F_{t}^{i} N_{t}^{-1}\left(F_{t}^{i}\right)^{\tau}\right]^{-1} \\
& \cdot {\left[K_{t} E_{t}^{i}-K_{t} F_{t}^{i} N_{t}^{-1} B_{t}^{\tau} K_{t}\right]-\sum_{i=1}^{\infty} K_{t} B_{t} N_{t}^{-1}\left(F_{t}^{i}\right)^{\tau} } \\
& \cdot {\left[I_{n}+\sum_{i=1}^{\infty} K_{t} F_{t}^{i} N_{t}^{-1}\left(F_{t}^{i}\right)^{\tau}\right]^{-1} K_{t} E_{t}^{i} } \\
&+\sum_{i=1}^{\infty} K_{t} B_{t} N_{t}^{-1}\left(F_{t}^{i}\right)^{\tau}\left[I_{n}+K_{t} F_{t}^{i} N_{t}^{-1}\left(F_{t}^{i}\right)^{\tau}\right]^{-1} \\
& \cdot K_{t} F_{t}^{i} N_{t}^{-1} B_{t}^{\tau} K_{t} \\
& K_{T}= Q \\
& I_{n}+\sum_{i=1}^{\infty} K_{t} F_{t}^{i} N_{t}^{-1}\left(F_{t}^{i}\right)^{\tau}>0
\end{aligned}
$$$$
(i=1,2,3, \ldots) .
$$

Compare (39) and (40); we can find that if we can prove $K_{t}$ the solution of (40), then

$$
\begin{aligned}
& M_{t}=K_{t} C_{t}, \\
& Y_{t}^{i}=\left[I_{n}+K_{t} F_{t}^{i} N_{t}^{-1}\left(F_{t}^{i}\right)^{\tau}\right]^{-1}\left[K_{t} E_{t}^{i}-K_{t} F_{t}^{i} N_{t}^{-1} B_{t}^{\tau} K_{t}\right]
\end{aligned}
$$

is the solution of the Riccati equation (39).
In the following, we will focus on the existence and uniqueness of solutions of (40). Firstly, let $S_{+}^{n}$ denote the space of all $n \times n$ nonnegative symmetric matrices, and $C\left([0, T] ; S_{+}^{n}\right)$ is a Banach space of $S_{+}^{n}$-valued continuous functions on $[0, T]$. We have the following uniqueness result.

Theorem 10. The Riccati equation (40) admits at most one solution $K_{t} \in C\left[0, T ; S_{+}^{n}\right]$.

Proof. Suppose $\widetilde{K}_{t} \in C\left[0, T ; S_{+}^{n}\right]$ satisfying $I_{n}+$ $\sum_{i=1}^{\infty} \widetilde{K}_{t} F_{t}^{i} N_{t}^{-1}\left(F_{t}^{i}\right)^{\tau}>0$ is another solution of (40). Let $\widehat{K}_{t}=K_{t}-\widetilde{K}_{t}$; then

$$
\begin{aligned}
-\dot{\widehat{K}}_{t} & \\
= & A_{t}^{\tau} \widehat{K}_{t}+\widehat{K}_{t} A_{t}+C_{t}^{\tau} \widehat{K}_{t} C_{t} \\
& \quad-\widehat{K}_{t}\left(L_{t}^{\tau}+B_{t} N_{t}^{-1} B_{t}^{\tau}\right) K_{t} \\
& \quad-\widetilde{K}_{t}\left(L_{t}^{\tau}+B_{t} N_{t}^{-1} B_{t}^{\tau}\right) \widehat{K}_{t}+I_{1}+I_{2}+I_{3}+I_{4} \\
& \widehat{K}_{T}= \\
& 0, \\
I_{n}+ & \sum_{i=1}^{\infty} \widehat{K}_{t} F_{t}^{i} N_{t}^{-1}\left(F_{t}^{i}\right)^{\tau}>0, \quad(i=1,2,3, \ldots),
\end{aligned}
$$

where

$$
\begin{aligned}
I_{1}= & \sum_{i=1}^{\infty}\left[\left(E_{t}^{i}\right)^{\tau}\left[I_{n}+K_{t} F_{t}^{i} N_{t}^{-1}\left(F_{t}^{i}\right)^{\tau}\right]^{-1} \widehat{K}_{t} E_{t}^{i}\right] \\
& -\sum_{i=1}^{\infty}\left[\left(E_{t}^{i}\right)^{\tau}\left[I_{n}+K_{t} F_{t}^{i} N_{t}^{-1}\left(F_{t}^{i}\right)^{\tau}\right]^{-1} \widehat{K}_{t} F_{t}^{i} N_{t}^{-1}\left(F_{t}^{i}\right)^{\tau}\right. \\
& \left.\cdot\left[I_{n}+\widetilde{K}_{t} F_{t}^{i} N_{t}^{-1}\left(F_{t}^{i}\right)^{\tau}\right]^{-1} \widetilde{K}_{t} E_{t}^{i}\right] \\
I_{2}= & \sum_{i=1}^{\infty}\left[\widehat{K}_{t} B_{t} N_{t}^{-1}\left(F_{t}^{i}\right)^{\tau}\left[I_{n}+K_{t} F_{t}^{i} N_{t}^{-1}\left(F_{t}^{i}\right)^{\tau}\right]^{-1}\right. \\
& \left.\cdot K_{t} F_{t}^{i} N_{t}^{-1} B_{t}^{\tau} K_{t}\right]+\sum_{i=1}^{\infty}\left[\widetilde{K}_{t} B_{t} N_{t}^{-1}\left(F_{t}^{i}\right)^{\tau}\right. \\
& \left.\cdot\left[I_{n}+K_{t} F_{t}^{i} N_{t}^{-1}\left(F_{t}^{i}\right)^{\tau}\right]^{-1} \widehat{K}_{t} F_{t}^{i} N_{t}^{-1} B_{t}^{\tau} K_{t}\right] \\
& +\sum_{i=1}^{\infty}\left[\widetilde{K}_{t} B_{t} N_{t}^{-1}\left(F_{t}^{i}\right)^{\tau}\left[I_{n}+K_{t} F_{t}^{i} N_{t}^{-1}\left(F_{t}^{i}\right)^{\tau}\right]^{-1}\right. \\
& \left.\cdot\left[I_{n}+\widetilde{K}_{t} F_{t}^{i} N_{t}^{-1}\left(F_{t}^{i}\right)^{\tau}\right]^{-1} \widetilde{K}_{t} F_{t}^{i} N_{t}^{-1} B_{t}^{\tau} \widetilde{K}_{t}\right] \\
& \left.\cdot\left[I_{n} F_{t}^{i} N_{t}^{-1} B_{t}^{\tau} \widehat{K}_{t}\right]-K_{t} F_{t}^{i} N_{t}^{-1}\left(F_{t}^{i}\right)^{\tau}\right]_{t}^{-1} \widehat{K}_{t} F_{t}^{i} N_{t}^{-1}\left(F_{t}^{i}\right)^{\tau} \\
& \\
&
\end{aligned}
$$




$$
\begin{aligned}
& I_{3}=-\sum_{i=1}^{\infty}\left[\left(E_{t}^{i}\right)^{\tau}\left[I_{n}+K_{t} F_{t}^{i} N_{t}^{-1}\left(F_{t}^{i}\right)^{\tau}\right]^{-1}\right. \\
& \left.\cdot \widehat{K}_{t} F_{t}^{i} N_{t}^{-1} B_{t}^{\tau} K_{t}\right]-\sum_{i=1}^{\infty}\left[\left(E_{t}^{i}\right)^{\tau}\right. \\
& \left.\cdot\left[I_{n}+K_{t} F_{t}^{i} N_{t}^{-1}\left(F_{t}^{i}\right)^{\tau}\right]^{-1} \widetilde{K}_{t} F_{t}^{i} N_{t}^{-1} B_{t}^{\tau} \widehat{K}_{t}\right] \\
& -\sum_{i=1}^{\infty}\left[\widehat{K}_{t} B_{t} N_{t}^{-1}\left(F_{t}^{i}\right)^{\tau}\left[I_{n}+K_{t} F_{t}^{i} N_{t}^{-1}\left(F_{t}^{i}\right)^{\tau}\right]^{-1}\right. \\
& \left.\cdot K_{t} E_{t}^{i}\right]-\sum_{i=1}^{\infty}\left[\widetilde{K}_{t} B_{t} N_{t}^{-1}\left(F_{t}^{i}\right)^{\tau}\right. \\
& \left.\cdot\left[I_{n}+K_{t} F_{t}^{i} N_{t}^{-1}\left(F_{t}^{i}\right)^{\tau}\right]^{-1} \widehat{K}_{t} E_{t}^{i}\right]+\sum_{i=1}^{\infty}\left[\left(E_{t}^{i}\right)^{\tau}\right. \\
& \cdot\left[I_{n}+K_{t} F_{t}^{i} N_{t}^{-1}\left(F_{t}^{i}\right)^{\tau}\right]^{-1} \widehat{K}_{t} F_{t}^{i} N_{t}^{-1}\left(F_{t}^{i}\right)^{\tau} \\
& \left.\cdot\left[I_{n}+\widetilde{K}_{t} F_{t}^{i} N_{t}^{-1}\left(F_{t}^{i}\right)^{\tau}\right]^{-1} \widetilde{K}_{t} F_{t}^{i} N_{t}^{-1} B_{t}^{\tau} \widetilde{K}_{t}\right] \\
& +\sum_{i=1}^{\infty}\left[\widetilde{K}_{t} B_{t} N_{t}^{-1}\left(F_{t}^{i}\right)^{\tau}\left[I_{n}+K_{t} F_{t}^{i} N_{t}^{-1}\left(F_{t}^{i}\right)^{\tau}\right]^{-1}\right. \\
& \left.\cdot \widehat{K}_{t} F_{t}^{i} N_{t}^{-1}\left(F_{t}^{i}\right)^{\tau}\left[I_{n}+\widetilde{K}_{t} F_{t}^{i} N_{t}^{-1}\left(F_{t}^{i}\right)^{\tau}\right]^{-1} \widetilde{K}_{t} E_{t}^{i}\right] \\
& I_{4}=-\sum_{i=1}^{\infty}\left[\widehat{K}_{t} B_{t} N_{t}^{-1}\left(F_{t}^{i}\right)^{\tau}\left[I_{n}+K_{t} F_{t}^{i} N_{t}^{-1}\left(F_{t}^{i}\right)^{\tau}\right]^{-1}\right. \\
& \left.\cdot K_{t} E_{t}^{i}\right]-\sum_{i=1}^{\infty}\left[\widetilde{K}_{t} B_{t} N_{t}^{-1}\left(F_{t}^{i}\right)^{\tau}\right. \\
& \left.\cdot\left[I_{n}+K_{t} F_{t}^{i} N_{t}^{-1}\left(F_{t}^{i}\right)^{\tau}\right]^{-1} \widehat{K}_{t} E_{t}^{i}\right] \\
& +\sum_{i=1}^{\infty}\left[\widetilde{K}_{t} B_{t} N_{t}^{-1}\left(F_{t}^{i}\right)^{\tau}\left[I_{n}+K_{t} F_{t}^{i} N_{t}^{-1}\left(F_{t}^{i}\right)^{\tau}\right]^{-1}\right. \\
& \left.\cdot \widehat{K}_{t} F_{t}^{i} N_{t}^{-1}\left(F_{t}^{i}\right)^{\tau}\left[I_{n}+\widetilde{K}_{t} F_{t}^{i} N_{t}^{-1}\left(F_{t}^{i}\right)^{\tau}\right]^{-1} \widetilde{K}_{t} E_{t}^{i}\right] .
\end{aligned}
$$

$\left[I_{n}+K_{t} F_{t}^{i} N_{t}^{-1}\left(F_{t}^{i}\right)^{\tau}\right]^{-1}$ and $\left[I_{n}+\widetilde{K}_{t} F_{t}^{i} N_{t}^{-1}\left(F_{t}^{i}\right)^{\tau}\right]^{-1}$ are uniformly bounded as they are continuously in [0,T]; apply Gronwall's inequality; we can get, for all $t \in[0, T], \widehat{K}_{t}=0$. Then we prove the uniqueness of solution.

For the existence part, first of all, if we let

$$
\Phi_{t}=\Lambda(K)=\left[I_{n}+\sum_{i=1}^{\infty} K F^{i} N^{-1}\left(F^{i}\right)^{\tau}\right]^{-1} K,
$$

then from the conventional Riccati equation theory, for all $\Phi_{t} \in C\left([0, T] ; S_{+}^{n}\right)$, the following conventional Riccati equation

$$
-\dot{K}_{t}=\left[A_{t}-\sum_{i=1}^{\infty} B_{t} N_{t}^{-1}\left(F_{t}^{i}\right)^{\tau} \Phi_{t} E_{t}^{i}\right]^{\tau} K_{t}+K_{t}\left[A_{t}\right.
$$

$$
\begin{aligned}
& \left.-\sum_{i=1}^{\infty} B_{t} N_{t}^{-1}\left(F_{t}^{i}\right)^{\tau} \Phi_{t} E_{t}^{i}\right]-K_{t}\left[L_{t}^{\tau}+B_{t} N_{t}^{-1} B_{t}^{\tau}\right. \\
& \left.-\sum_{i=1}^{\infty} B_{t} N_{t}^{-1}\left(F_{t}^{i}\right)^{\tau} \Phi_{t} F_{t}^{i} N_{t}^{-1} B_{t}^{\tau}\right] K_{t}+C_{t}^{\tau} K_{t} C_{t} \\
& +\sum_{i=1}^{\infty}\left(E_{t}^{i}\right)^{\tau} \Phi_{t} E_{t}^{i}+R_{t} \\
K_{T}= & Q, \\
I_{n}+\sum_{i=1}^{\infty} K_{t} F_{t}^{i} N_{t}^{-1}\left(F_{t}^{i}\right)^{\tau}>0, & (i=1,2,3, \ldots) .
\end{aligned}
$$

has a unique solution $K(\cdot) \in C\left([0, T] ; S_{+}^{n}\right)$, when

$$
\begin{aligned}
& {\left[L_{t}^{\tau}+B_{t} N_{t}^{-1} B_{t}^{\tau}-\sum_{i=1}^{\infty}\left[B_{t} N_{t}^{-1}\left(F_{t}^{i}\right)^{\tau} \Phi F_{t}^{i} N_{t}^{-1} B_{t}\right]\right]} \\
& \quad \in C\left([0, T] ; S_{+}^{n}\right) .
\end{aligned}
$$

Let $S_{s}^{n}$ be the subspace of $S_{+}^{n}$ which is formed by the symmetric matrices satisfying (46). Obviously, as $K_{t} \equiv 0 \epsilon$ $S_{s}^{n}$ the definition of $S_{s}^{n}$ is reasonable. Define a mapping $\Psi$ : $C\left([0, T] ; S_{s}^{n}\right) \rightarrow C\left([0, T] ; S_{+}^{n}\right)$; we can get Lemma 11 about $\Phi$ and $\Psi$.

Lemma 11. The operators $\Phi=\Lambda(K)$ are monotonously increasing when $K>0$, and the operator $\Psi$ is continuous and monotonously increasing.

Proof. When $K>0$, from the definition of $\Lambda(K)$, we have

$$
\begin{aligned}
\Lambda(K) & =\left[I_{n}+\sum_{i=1}^{\infty} K F^{i} N^{-1}\left(F^{i}\right)^{\tau}\right]^{-1} K \\
& =\left[K^{-1}\left(I_{n}+\sum_{i=1}^{\infty} K F^{i} N^{-1}\left(F^{i}\right)^{\tau}\right)\right]^{-1} \\
& =\left[K^{-1}+\sum_{i=1}^{\infty} F^{i} N^{-1}\left(F^{i}\right)^{\tau}\right]^{-1} .
\end{aligned}
$$

So if $K_{1} \geq K_{2}$, then $\Lambda\left(K_{1}\right) \geq \Lambda\left(K_{2}\right)$; that is, $\Lambda(K)$ is monotonously increasing when $K>0$.

As $K=\Psi(\Phi)$, set $\bar{K}=\Psi(\bar{\Phi})$; then the conventional Riccati equation (45) can be rewritten

$$
\begin{aligned}
& -\dot{K}_{t} \\
& =A_{t}^{\tau} K_{t}+K_{t} A_{t}-K_{t}\left[L_{t}^{\tau}+B_{t} N_{t}^{-1} B_{t}^{\tau}\right] K_{t}+R_{t}
\end{aligned}
$$




$$
\begin{aligned}
& +C_{t}^{\tau} K_{t} C_{t} \\
& +\sum_{i=1}^{\infty}\left[\left[E_{t}^{i}-F_{t}^{i} N_{t}^{-1} B_{t}^{\tau} K_{t}\right]^{\tau} \Phi_{t}\left[E_{t}^{i}-F_{t}^{i} N_{t}^{-1} B_{t}^{\tau} K_{t}\right]\right] \\
K_{T}= & Q
\end{aligned}
$$

$$
(i=1,2,3, \ldots) \text {. }
$$

From the conclusion of $\Lambda(K)$ above in this lemma and Lemma 8.2 in [28], if $\Phi \geq \bar{\Phi}$ then $K \geq \bar{K}$; the operator $\Psi$ is monotonously increasing. On the other hand, by Gronwall's inequality, we know that if $\Phi \rightarrow \bar{\Phi}$, then $K-\bar{K} \rightarrow 0$, so the operator $\Psi$ is also continuous.

For (45), it is easy to know that if there exists $\Phi(\cdot) \in$ $C\left([0, T] ; S_{+}^{n}\right)$ satisfying

$$
\Phi=\left[I_{n}+\sum_{i=1}^{\infty} \Psi(\Phi) F^{i} N^{-1}\left(F^{i}\right)^{\tau}\right]^{-1} \Psi(\Phi),
$$

then Riccati equation (40) admits a unique solution. So the following task is to find the suitable $\Phi(\cdot) \in C\left([0, T] ; S_{+}^{n}\right)$ satisfying (49). We need the following lemma.

Lemma 12. If there exist $\Phi^{+}, \Phi^{-} \in C\left([0, T] ; S_{s}^{n}\right)$ which satisfy

$$
\begin{aligned}
\Phi^{+} & \geq\left[I_{n}+\sum_{i=1}^{\infty} \Psi\left(\Phi^{+}\right) F^{i} N^{-1}\left(F^{i}\right)^{\tau}\right]^{-1} \Psi\left(\Phi^{+}\right) \\
& \geq\left[I_{n}+\sum_{i=1}^{\infty} \Psi\left(\Phi^{-}\right) F^{i} N^{-1}\left(F^{i}\right)^{\tau}\right]^{-1} \Psi\left(\Phi^{-}\right) \geq \Phi^{-},
\end{aligned}
$$

then Riccati equation (40) admits a solution $K(\cdot) \in C([0, T]$; $\left.S_{+}^{n}\right)$.

Proof. For given $\Phi^{+}, \Phi^{-}$which satisfied (49), define the sequences $\Phi_{j}^{+}, \Phi_{j}^{-}, K_{j}^{+}, K_{j}^{-}$as follows:

$$
\begin{aligned}
\Phi_{0}^{+} & =\Phi^{+} \in S_{s}^{n}, \\
\Phi_{0}^{-} & =\Phi^{-} \in S_{s}^{n}, \\
K_{0}^{+} & =\Psi\left(\Phi_{0}^{+}\right), \\
K_{0}^{-} & =\Psi\left(\Phi_{0}^{-}\right), \\
\Phi_{j+1}^{+} & =\left[I_{n}+\sum_{i=1}^{\infty} K F^{i} N^{-1}\left(F^{i}\right)^{\tau}\right]^{-1} K_{j}^{+}, \\
\Phi_{j+1}^{-} & =\left[I_{n}+\sum_{i=1}^{\infty} K F^{i} N^{-1}\left(F^{i}\right)^{\tau}\right]^{-1} K_{j}^{-}, \\
K_{j+1}^{+} & =\Psi\left(\Phi_{j+1}^{+}\right), \\
K_{j+1}^{-} & =\Psi\left(\Phi_{j+1}^{-}\right),
\end{aligned}
$$

From (50) and Lemma 11, by induction, we obtain

$$
\begin{aligned}
& K_{0}^{+} \geq K_{j}^{+} \geq K_{j+1}^{+} \geq K_{j+1}^{-} \geq K_{j}^{-} \geq K_{0}^{-} \geq 0, \\
& \Phi_{0}^{+} \geq \Phi_{j}^{+} \geq \Phi_{j+1}^{+} \geq \Phi_{j+1}^{-} \geq \Phi_{j}^{-} \geq \Phi_{0}^{-} \geq 0,
\end{aligned}
$$

and $\Phi_{j}^{+}, \Phi_{j}^{-} \in S_{s}^{n}$; we have

$$
\begin{aligned}
\lim _{j \rightarrow \infty} \Phi_{j}^{+} & =\Phi^{+} \in S_{s}^{n}, \\
\lim _{j \rightarrow \infty} K_{j}^{+} & =K^{+} \in S_{+}^{n}, \\
K^{+} & =\lim _{j \rightarrow \infty} K_{j}^{+}=\lim _{j \rightarrow \infty} \Psi\left(\Phi_{j}^{+}\right)=\Psi\left(\lim _{j \rightarrow \infty} \Phi_{j}^{+}\right) \\
& =\Psi\left(\Phi_{j}^{+}\right) .
\end{aligned}
$$

So $K^{+}$is a solution of (45) corresponding to $\Phi=\Phi^{+}$; then

$$
\Phi^{+}=\left[I_{n}+\sum_{i=1}^{\infty} K F^{i} N^{-1}\left(F^{i}\right)^{\tau}\right]^{-1} K^{+},
$$

where $K^{+}$is a solution of Riccati equation (40). By the same step, we can get

$$
\begin{aligned}
& \lim _{j \rightarrow \infty} \Phi_{j}^{-}=\Phi^{-} \in S_{s}^{n}, \\
& \lim _{j \rightarrow \infty} K_{j}^{-}=K^{-} \in S_{+}^{n} .
\end{aligned}
$$

and $K^{-}$is also a solution of Riccati equation (40). From Theorem $10, K^{+}=K^{-}$.

From Lemma 12, in order to get the existence of solution for Riccati equation (40), we only need to find $\Phi^{+}$and $\Phi^{-}$ satisfying (50). Obviously, we can let $\Phi^{-}=0$ and for the existence of $\Phi^{+}$, we need the following Assumption 13.

Assumption 13. There exists $\bar{\Phi}(\cdot) \in S_{s}^{n}$, such that

$$
\begin{aligned}
\sum_{i=1}^{\infty}\left(F^{i}\right)^{\tau}(t) \bar{\Phi}(t) F^{i}(t) & =N(t), \\
\sum_{i=1}^{\infty}\left[I_{n}+\bar{K}(t) F^{i}(t) N^{-1}\left(F^{i}\right)^{\tau}(t)\right]^{-1} \bar{K}(t) & \leq \bar{\Phi}(t),
\end{aligned}
$$

where $\bar{K}(t)$ is the unique solution of the following equation:

$$
\begin{aligned}
-\dot{\bar{K}}_{t}= & {\left[A_{t}-\sum_{i=1}^{\infty} B_{t} N_{t}^{-1}\left(F_{t}^{i}\right)^{\tau} \bar{\Phi}(t) E_{t}^{i}\right]^{\tau} \bar{K}_{t} } \\
& +\bar{K}_{t}\left[A_{t}-\sum_{i=1}^{\infty} B_{t} N_{t}^{-1}\left(F_{t}^{i}\right)^{\tau} \bar{\Phi}(t) E_{t}^{i}\right] \\
& -\bar{K}_{t} L_{t}^{\tau} \bar{K}_{t}+C_{t}^{\tau} \bar{K}_{t} C_{t}+\sum_{i=1}^{\infty}\left(E_{t}^{i}\right)^{\tau} \bar{\Phi}(t) E_{t}^{i} \\
& +R_{t} \\
\bar{K}_{T}= & Q .
\end{aligned}
$$

$$
j=1,2,3, \ldots
$$


It is easy to known that when matrix $F_{t}$ is invertible and $k=n$, Assumption 13 is satisfied. Then we get the main result of this section.

Theorem 14. Let $D=0$ and Assumption 13 holds; the Riccati equation (40) has a unique solution $(K, M, Y) \in$ $C^{1}\left([0, T] ; S_{+}^{n}\right) \times L^{\infty}\left([0, T] ; R^{n \times n}\right) \times L^{\infty}\left([0, T] ; R^{n \times n}\right)$.

At last, we give a simple example of the Riccati equation which has a unique solution.

Example 15. Assume the dimensions of the state and control are the same; that is, $k=n$, when $D=0, F^{i}=I_{n}, \quad(i=$ $1,2,3, \ldots)$ in Riccati equation (39); let $\bar{\Phi}(\cdot)=N(\cdot)$; then check Assumption 13.

In fact, $\bar{\Phi}(\cdot)=N(\cdot) \geq 0$, so $\sum_{i=1}^{\infty}\left[\bar{\Phi}_{t} F_{t}^{i}+\right.$ $\left.\bar{K}_{t} F_{t}^{i} N_{t}^{-1}\left(F_{t}^{i}\right)^{\tau} \bar{\Phi}_{t} F_{t}^{i}\right] \geq \sum_{i=1}^{\infty} \bar{K}_{t} F_{t}^{i}$; here $\bar{K}_{t}$ is the solution of

$$
\begin{aligned}
-\dot{\bar{K}}_{t}= & {\left[A_{t}-\sum_{i=1}^{\infty} B_{t} E_{t}^{i}\right]^{\tau} \bar{K}_{t}+\bar{K}_{t}\left[A_{t}-\sum_{i=1}^{\infty} B_{t} E_{t}^{i}\right] } \\
& -\bar{K}_{t} L_{t}^{\tau} \bar{K}_{t}+C_{t}^{\tau} \bar{K}_{t} C_{t}+\sum_{i=1}^{\infty}\left(E_{t}^{i}\right)^{\tau} N(t) E_{t}^{i} \\
& +R_{t} \\
\bar{K}_{T}= & Q .
\end{aligned}
$$

$\bar{\Phi}_{t}+\sum_{i=1}^{\infty} \bar{K}_{t} F_{t}^{i} N_{t}^{-1}\left(F_{t}^{i}\right)^{\tau} \bar{\Phi}_{t} \geq \bar{K}_{t}$, and then $\left[I_{n}+\right.$ $\left.\sum_{i=1}^{\infty} \bar{K}_{t} F_{t}^{i} N_{t}^{-1}\left(F_{t}^{i}\right)^{\tau}\right]^{-1} \bar{K}_{t} \leq \bar{\Phi}_{t}$. From Theorem 14, the Riccati equation (39) has a unique solution when $k=n, D=0, F^{i}=$ $I_{n},(i=1,2,3, \ldots)$.

\section{Conclusion}

In this paper, we discussed one kind of LQ stochastic control problem with Lévy process as noise source where the control system is described by a linear FBSDEL. Explicit form of optimal control is obtained, and it can be proved to be unique. When assuming that all the coefficient matrices in this control problem are deterministic, it has been shown that the linear feedback regulator for this LQ problem has a close relation to the solutions of a kind of generalized Riccati equation. Finally, we discuss the solvability of the generalized Riccati equation and prove the existence and uniqueness of the solution for it in a special case.

\section{Conflicts of Interest}

The authors declare that they have no conflicts of interest.

\section{Acknowledgments}

This work was supported by the National Natural Science Foundation of China (no. 11271007, no. 61673013), the Natural Science Foundation of Shandong Province (no. ZR2016JZ022), the SDUST Research Fund (no.
2014JQJH103), and the Graduate Student Innovation Fund of SDUST (no. SDKDYC170345).

\section{References}

[1] H. Kushner, "Optimal Stochastic Control," IRE Transactions on Automatic Control, vol. 7, no. 5, pp. 120-122, 1962.

[2] W. M. Wonham, "On a matrix Riccati equation of stochastic control," SIAM Journal on Control and Optimization, vol. 6, pp. 681-697, 1968.

[3] G. Li and W. Zhang, "Study on indefinite stochastic linear quadratic optimal control with inequality constraint," Journal of Applied Mathematics, vol. 2013, Article ID 805829, 9 pages, 2013.

[4] H.-j. Ma and T. Hou, "A separation theorem for stochastic singular linear quadratic control problem with partial information," Acta Mathematicae Applicatae Sinica. English Series, vol. 29, no. 2, pp. 303-314, 2013.

[5] X. Liu, Y. Li, and W. Zhang, "Stochastic linear quadratic optimal control with constraint for discrete-time systems," Applied Mathematics and Computation, vol. 228, pp. 264-270, 2014.

[6] X. Wang, X. Zhang, and P. Zhao, "Binary nonlinearization for AKNS-KN coupling system," Abstract and Applied Analysis, Article ID 253102, 12 pages, 2014.

[7] C. H. Shen and X. R. Wang, "Nonlinear analysis on the pattern structures of connection between final markets," Journal of Management sciences in China, vol. 18, no. 2, pp. 66-75, 2015.

[8] X.-R. Wang, Z.-Y. Gao, and Z. Wu, "Forward-backward stochastic differential equation and the linear quadratic stochastic optimal control," Acta Automatica Sinica, vol. 29, no. 1, pp. 3237, 2003.

[9] Z. Wu, "Forward-backward stochastic differential equations, linear quadratic stochastic optimal control and nonzero sum differential games," Journal of Systems Science and Complexity, vol. 2, pp. 179-192, 18.

[10] R. Boel and P. Varaiya, "Optimal control of jump processes," SIAM Journal on Control and Optimization, vol. 15, no. 1, pp. 92-119, 1977.

[11] Z. Wu and X. Wang, "FBSDE with Poisson process and its application to linear quadratic stochastic optimal control problem with random jumps," Acta Automatica Sinica, vol. 29, no. 6, pp. 821-826, 2003.

[12] J. T. Shi and Z. Wu, "One kind of fully coupled linear quadratic stochastic control problem with random jumps," Acta Automatica Sinica. Zidonghua Xuebao, vol. 35, no. 1, pp. 92-97, 2009.

[13] X. Lin and R. Zhang, "Ho control for stochastic systems with Poisson jumps," Journal of Systems Science \& Complexity, vol. 24, no. 4, pp. 683-700, 2011.

[14] X. Li, X. Lin, and Y. Lin, "Lyapunov-type conditions and stochastic differential equations driven by G-Brownian motion," Journal of Mathematical Analysis and Applications, vol. 439, no. 1, pp. 235-255, 2016.

[15] D. Nualart and W. Schoutens, "Chaotic and predictable representations for Lévy processes," Stochastic Processes and their Applications, vol. 90, no. 1, pp. 109-122, 2000.

[16] D. Nualart and W. Schoutens, "Backward stochastic differential equations and Feynman-Kac formula for Lévy processes, with applications in finance," Bernoulli. Official Journal of the Bernoulli Society for Mathematical Statistics and Probability, vol. 7, no. 5, pp. 761-776, 2001. 
[17] K. Bahlali, M. Eddahbi, and E. Essaky, "BSDE associated with Lévy processes and application to PDIE," Journal of Applied Mathematics and Stochastic Analysis, vol. 16, no. 1, pp. 1-17, 2003.

[18] M. El Otmani, "Generalized BSDE driven by a Lévy process," Journal of Applied Mathematics and Stochastic Analysis, Art. ID 85407, 25 pages, 2006.

[19] M. El Otmani, "Backward stochastic differential equations associated with Lévy processes and partial integro-differential equations," Communications on Stochastic Analysis, vol. 2, no. 2, pp. 277-288, 2008.

[20] Y. Ren and X. Fan, "Reflected backward stochastic differential equations driven by a lvy process," ANZIAM Journal, vol. 50, no. 4, pp. 486-500, 2009.

[21] M. Tang and Q. Zhang, "Optimal variational principle for backward stochastic control systems associated with Lévy processes," Science China. Mathematics, vol. 55, no. 4, pp. 745-761, 2012.

[22] H. Huang and X. Wang, "LQ stochastic optimal control of forward-backward stochastic control system driven by Lévy process," in Proceedings of the 2016 IEEE Advanced Information Management, Communicates, Electronic and Automation Control Conference, IMCEC 2016, pp. 1939-1943, chn, October 2016.

[23] K.-i. Mitsui and Y. Tabata, "A stochastic linear-quadratic problem with Lévy processes and its application to finance," Stochastic Processes and their Applications, vol. 118, no. 1, pp. 120152, 2008.

[24] H. Tang and Z. Wu, "Stochastic differential equations and stochastic linear quadratic optimal control problem with Lévy processes," Journal of Systems Science \& Complexity, vol. 22, no. 1, pp. 122-136, 2009.

[25] R. S Pereira and E. Shamarova, "Forward backward SDEs driven by Lévy processes and application to option pricing," Random Operators and Stochastic Equations, vol. 2, no. 1, pp. 1-20, 2012.

[26] F. Baghery, N. Khelfallah, B. Mezerdi, and I. Turpin, "Fully coupled forward backward stochastic differential equations driven by Lévy processes and application to differential games," Random Operators and Stochastic Equations, vol. 22, no. 3, pp. 151-161, 2014.

[27] X. Wang and H. Huang, "Maximum principle for forwardbackward stochastic control system driven by Lévy process," Mathematical Problems in Engineering, Article ID 702802, Art. ID 702802, 12 pages, 2015.

[28] S. Peng, "Problem of eigenvalues of stochastic Hamiltonian systems with boundary conditions," Stochastic Processes and their Applications, vol. 88, no. 2, pp. 259-290, 2000. 


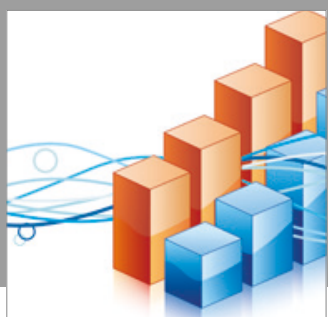

Advances in

Operations Research

vatersals

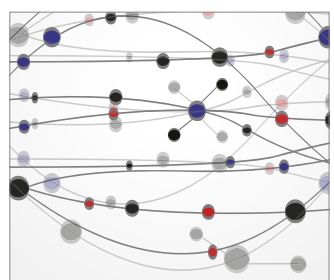

\section{The Scientific} World Journal
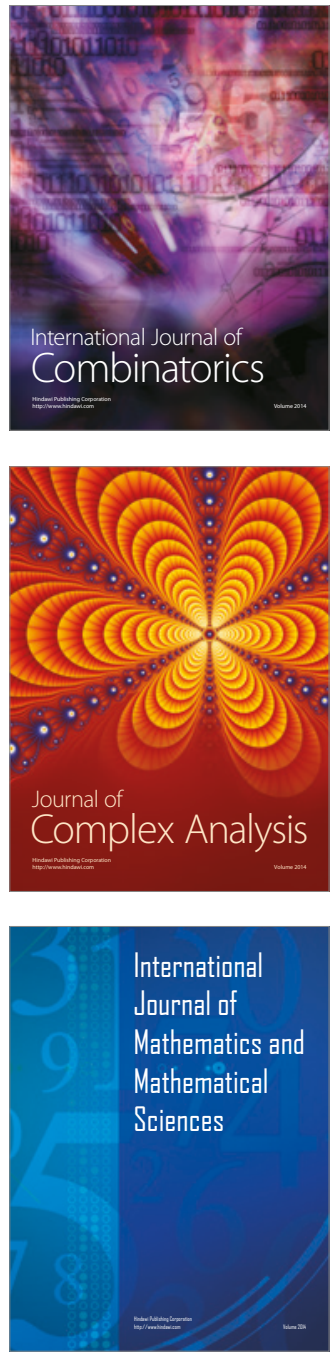
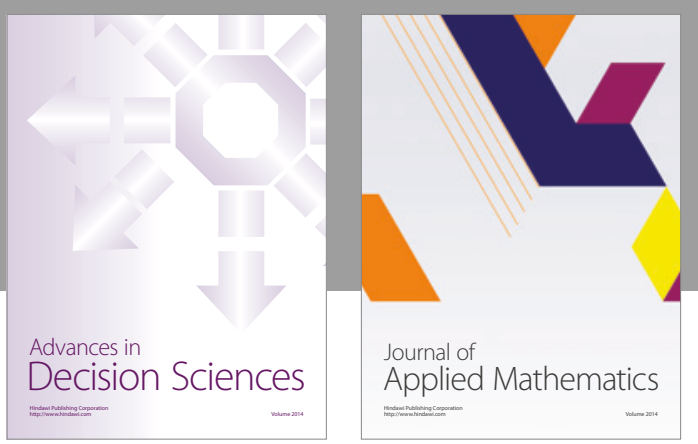

Algebra

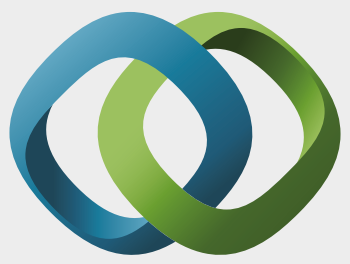

\section{Hindawi}

Submit your manuscripts at

https://www.hindawi.com
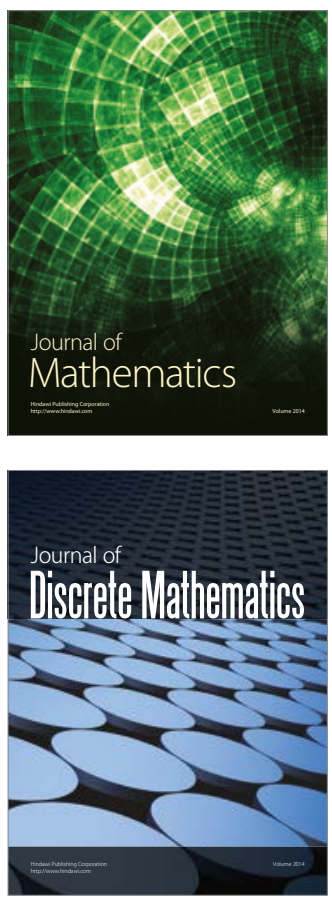

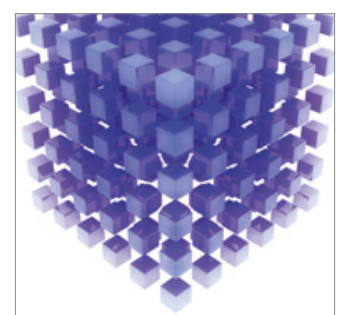

Mathematical Problems in Engineering
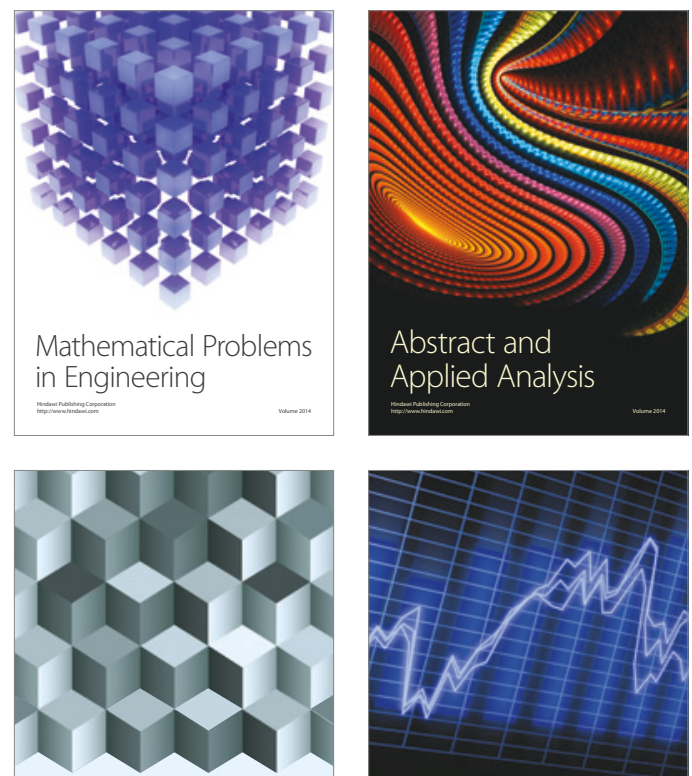

Journal of

Function Spaces

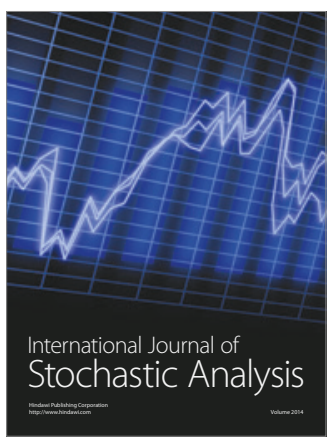

Probability and Statistics
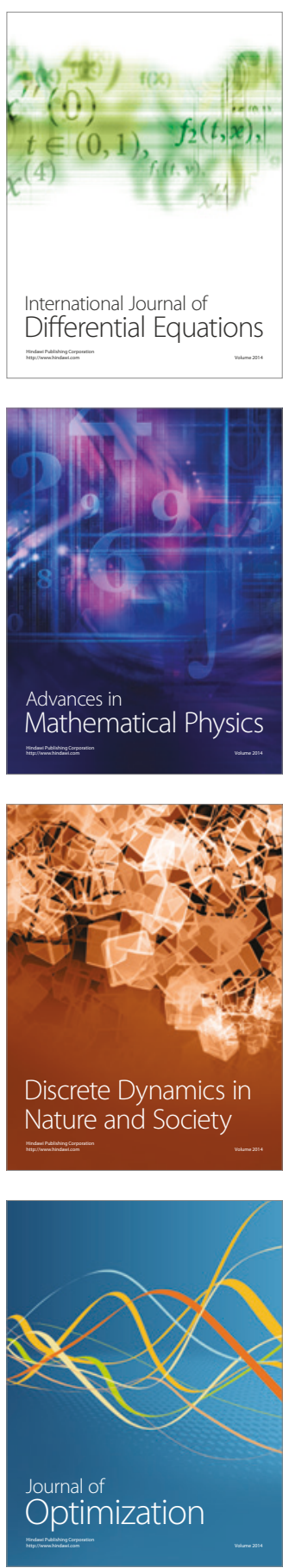